\title{
Topology optimization for transient response of photonic crystal structures
}

\author{
Matzen, René; Jensen, Jakob Søndergaard; Sigmund, Ole
}

Published in:

Optical Society of America. Journal B: Optical Physics

Link to article, DOI:

10.1364/JOSAB.27.002040

Publication date:

2010

Document Version

Publisher's PDF, also known as Version of record

Link back to DTU Orbit

Citation (APA):

Matzen, R., Jensen, J. S., \& Sigmund, O. (2010). Topology optimization for transient response of photonic crystal structures. Optical Society of America. Journal B: Optical Physics, 27(10), 2040-2050.

https://doi.org/10.1364/JOSAB.27.002040

\section{General rights}

Copyright and moral rights for the publications made accessible in the public portal are retained by the authors and/or other copyright owners and it is a condition of accessing publications that users recognise and abide by the legal requirements associated with these rights.

- Users may download and print one copy of any publication from the public portal for the purpose of private study or research.

- You may not further distribute the material or use it for any profit-making activity or commercial gain

- You may freely distribute the URL identifying the publication in the public portal

If you believe that this document breaches copyright please contact us providing details, and we will remove access to the work immediately and investigate your claim. 


\title{
Topology optimization for transient response of photonic crystal structures
}

\author{
René Matzen,* Jakob S. Jensen, and Ole Sigmund \\ Department of Mechanical Engineering, Technical University of Denmark, Nils Koppels Allé, Building 404, \\ 2800 Kgs. Lyngby, Denmark \\ *Corresponding author: rmat@mek.dtu.dk
}

Received June 22, 2010; accepted August 4, 2010;

posted August 13, 2010 (Doc. ID 130543); published September 16, 2010

\begin{abstract}
An optimization scheme based on topology optimization for transient response of photonic crystal structures is developed. The system response is obtained by a finite-element time-domain analysis employing perfectly matched layers as an absorbing boundary condition. As an example a waveguide-side-coupled microcavity is designed. The gradient-based optimization technique is applied to redistribute the material inside the microcavity such that the $Q$ factors of a monopole and a dipole mode are improved by $375 \%$ and $285 \%$, respectively, while maintaining strong coupling. This is obtained by maximizing the stored energy inside the microcavity in the decaying regime of the transient response. Manufacturable designs are achieved by filtering techniques capable of controlling minimum length scales of the design features. (C) 2010 Optical Society of America

OCIS codes: $000.4430,230.5750,230.7390$.
\end{abstract}

\section{INTRODUCTION}

In the field of information technology light plays a progressively important role as an information conveyor. One of the keys to future information technology is the realization of large-scale optical integrated circuits. Herein photonic crystals (PhCs) are believed to be the leading platforms. This is due to their unique capability of exhibiting very strong light-matter interaction while keeping the size of the optical components greatly reduced [1,2]. It is the photonic bandgaps of $\mathrm{PhCs}$ that yield this interaction in which wave propagation at certain wavelengths is prohibited. The introduction of $\mathrm{PhC}$ mirrors, waveguides (WGs), and resonant microcavitites (MCs) as the three basic PhC components has led to an increased attention on the design of optical devices within the last decade that may accomplish the complete control of light propagation. Amalgamation of these components has been utilized to designing various $\mathrm{PhC}$ devices such as filters, bends, and splitters for various applications [3].

In the engineering of $\mathrm{PhC}$ devices, the use of inverse problem techniques has recently been introduced as potential designing tools to replace previous approaches $[4,5]$. In this paper we demonstrate how topology optimization based on transient analysis can be used to tweak the performance of a two-dimensional (2D) $\mathrm{PhC}$ filtering device, consisting of a PhC-WG-side-coupled PhC-MC. The aim is to achieve strong coupling between the $\mathrm{PhC}-\mathrm{WG}$ and -MC while maintaining a high $Q$ factor for the filtered mode residing inside the MC.

The methodology behind topology optimization has previously disclosed its usefulness in the design of various 2D PhC-WG components, e.g., a $90^{\circ}$ bend [6], a T-junction [7], and a termination [8]. Other relevant and efficient $\mathrm{PhC}$ component designs have been obtained and experimentally verified in [9-12]. The common goal for these optimization examples is the maximization of power trans- mission of either transverse electric (TE-) or transverse magnetic (TM)-polarized waves at multiple frequencies. The optimized components reveal good performance and agreement with experiments. The basis for the computational model is the finite-element frequency-domain method. A consequence of using frequency-domain methods is that wideband optimization requires multiple frequency analyses.

In the present paper the finite-element time-domain (FETD) method will be employed. Time-domain methods have the advantage of computing the response of a linear system at many frequencies with a single time-domain analysis. This idea has been used for antenna design using the finite-difference time-domain method [13], onedimensional filter and pulse modulator designs [14,15], and simultaneous space-time optimization [16] in the setting of the FETD method. Additionally, time-domain methods can accommodate strongly nonlinear or active (time-varying) media, whereas frequency methods have difficulties with these physical regimes because the frequency is no longer preserved. Two of the major challenges of the FETD method are the computational cost associated with the computation of the sensitivities and the implementation of efficient absorbing boundary conditions (ABCs), such as the perfectly matched layer (PML) [17]. To the authors' knowledge, a topology optimization scheme based on the FETD method using PMLs as ABCs has not been reported before.

Improving $Q$ factors of MCs has previously been studied and subjected to optimization in a $2 \mathrm{D} \mathrm{PhC} \mathrm{slab} \mathrm{with} \mathrm{a}$ triangular lattice pattern of air holes. These studies reveal that small mode volume and high $Q$ factors are essential in the realization of high-performing active lightemitting devices such as zero-threshold lasers. Standard designs of PhC-MCs exploit simple circular defects. A general recipe relying on an analytically derived inverse 
problem approach is developed in [18] restricted to geometries of circular shape. Alternatively, a more general inverse systematic approach based on a level-set approach for shape optimization is suggested in [19] that yields optimized designs of arbitrary convex shapes. Meanwhile, when considering the MC as side-coupled to a WG in filtering devices, it acts as a passive component. Consequently, the requirement for the mode volume becomes secondary, and the interaction between the $\mathrm{PhC}-\mathrm{MC}$ and the $\mathrm{PhC}-\mathrm{WG}$ is the primary target [20]. A detailed conceptual study of this interaction can be found in [21,22].

The purpose of this work is to utilize the free material distribution technique provided by topology optimization [23], and thereby not limiting the optimized design to any particular geometrical shape. The optimization method is formulated in the framework of the FETD method for TEpolarized modes, and it uses PMLs as ABCs (Section 2). We express the problem in a fashion that improves the $Q$ factor by maximizing the stored magnetic energy in the exponentially decaying regime of the transient response of $\mathrm{PhC}-\mathrm{MC}$ monopole and dipole modes. In the optimization process we consider a $2 \mathrm{D} \mathrm{PhC}$ configuration with a triangular lattice of air holes in the dielectric GaAs (Section 3). The system is homogenous in the third dimension, whereby the total $Q$ factor only depends on the in-plane $Q$ factor. However, the method presented here is believed also to be valid for optimization of the $Q$ factor for equivalent $\mathrm{PhC}$ slab devices, in which the out-of-plane $Q$ factor limits the total $Q$ factor. It can also immediately be adapted to TM modes. Finally (Section 4) by using temporal coupled-mode (CM) theory [24], the PhC filter with the optimized $\mathrm{PhC}-\mathrm{MC}$ is analyzed to verify that the spectral performance of the filter is improved as desired.

\section{FORMULATION OF THE TRANSIENT TOPOLOGY OPTIMIZATION METHOD}

Throughout this paper we consider propagation of TE modes within $2 \mathrm{D}$ infinitely tall $\mathrm{PhC}$ structures. The medium inside the structures is assumed to be invariant in time and to occupy a composite of regions of a homogeneous dielectric material as a function of the plane (Cartesian) position vector $\mathbf{r}=(x, y)$ in the solution domain $\Omega_{S}$. Due to the infinite extension in the third dimension, it is sufficient to solve the scalar wave equation for the transverse component of the magnetic field, $H_{z}(\mathbf{r}, t)$. The numerical solution is sought by truncating $\Omega_{S}$ with a PML region $\Omega_{\mathrm{PML}}$ as a means to minimize the nonphysical reflection from the boundary $\partial \Omega_{S}$ (see Fig. 1). Interpreting the material behavior in $\Omega_{\mathrm{PML}}$ as anisotropic, dispersive, and lossy, the governing equation for $\mathbf{r} \in \Omega=\Omega_{S} \cup \Omega_{\mathrm{PML}}$ takes the following general form [17]:

$\mu \mathcal{L}_{1}(t) H_{z}+\left[\frac{\partial}{\partial x}\left(\frac{\mathcal{L}_{2, x}(t)}{\varepsilon} \frac{\partial}{\partial x}\right)+\frac{\partial}{\partial y}\left(\frac{\mathcal{L}_{2, y}(t)}{\varepsilon} \frac{\partial}{\partial y}\right)\right] H_{z}=-\frac{\partial J_{B, z}}{\partial t}$,

where $\varepsilon=\varepsilon(\mathbf{r})$ and $\mu=\mu(\mathbf{r})$ denote permittivity and permeability, respectively.

On the right-hand side of Eq. (1), $J_{B, z}(\mathbf{r}, t)$ is the magnetic charge current, serving here as the excitation term

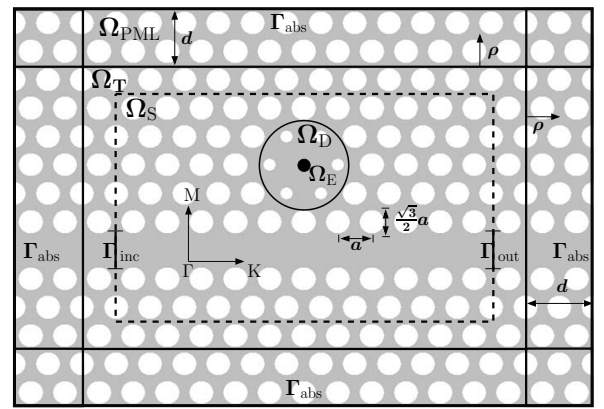

Fig. 1. Illustration of a $\Gamma-K$ directional PhC-WG structure as the computational domain. It consists of the solution domain $\Omega_{S}$ and the transition domain $\Omega_{T}$ that are truncated by the PML region $\Omega_{\mathrm{PML}}$. The circle encloses the scattering design region $\Omega_{D}$. In $\Omega_{E}$ (black circular region) the energy is maximized. The $\mathrm{PhC}$ is built by blocks of size $a / 2 \times \sqrt{3} a / 2$, where $\Omega_{T} \cup \Omega_{S}$ contains 30 $\times 14$ building blocks, and $\Omega_{\mathrm{PML}}$ is extended with 24 and 4 blocks on both sides in directions $\Gamma-K$ and $\Gamma-M$, respectively.

in the case of a radiating source residing in $\Omega_{S}$. The operator $\mathcal{L}_{1}(t)$ in Eq. (1) is given by

$$
\mathcal{L}_{1}(t)=\frac{\partial^{2}}{\partial t^{2}}+\frac{\sigma_{x}+\sigma_{y}}{\varepsilon} \frac{\partial}{\partial t}+\frac{\sigma_{x} \sigma_{y}}{\varepsilon^{2}}
$$

and the other operator $\mathcal{L}_{2, p}(t)$ is given by

$$
\mathcal{L}_{2, p}(t)=1-a_{p} \exp \left[-b_{p} t\right] \bar{u}(t) *, \quad p=x, y,
$$

with $a_{x}=\left(\sigma_{x}-\sigma_{y}\right) / \varepsilon, a_{y}=\left(\sigma_{y}-\sigma_{x}\right) / \varepsilon$, and $b_{p}=\sigma_{p} / \varepsilon$. In Eq. (3), $\bar{u}(t)$ denotes the Heaviside step function, and $*$ stands for temporal convolution. The spatially dependent coordinate-wise conductivities $\sigma_{x}$ and $\sigma_{y}$ attenuate the field in $\Omega_{\mathrm{PML}}$ and are thus only nonzero in $\Omega_{\mathrm{PML}}$. They are expressed in terms of the PML profile $\sigma(r)$ that is chosen here as [25]

$$
\begin{gathered}
\sigma(r)=\sigma_{\max }\left(\frac{\rho}{d}\right)^{m}, \\
\sigma_{\max }=-\frac{(m+1) \log _{10}\left(R_{0}\right)}{2 d Z_{0}} .
\end{gathered}
$$

In Eq. (4), $\rho$ denotes the perpendicular distance from the PML interface, $d$ is the width of the PML, $m$ is the order of the PML profile, $R_{0}$ is the theoretical reflection coefficient at normal incidence (typically around the order of $10^{-5}$ ), and $Z_{0}$ is the free-space material impedance. The impedance matching condition between $\Omega_{S}$ and $\Omega_{\mathrm{PML}}$ is satisfied by requiring that the permittivity in $\Omega_{\mathrm{PML}}$ [cf. Eqs. (2) and (3)] is determined by the value at the PML interface (i.e., when $\rho=0$ ) $[25,26]$. For convenience it is designated by $\varepsilon_{i}=\varepsilon(\rho=0)$.

If the PML is backed with the first-order Silver-Müller radiation boundary condition [27] on $\Gamma_{\text {abs }} \subseteq \partial \Omega$,

$$
\mathbf{n} \cdot\left(\varepsilon^{-1} \widetilde{\nabla} H_{z}\right)-Y \frac{\partial H_{z}}{\partial t}=0 \text { on } \Gamma_{\mathrm{abs}},
$$

where $\mathbf{n}$ is the outward unit vector normal to the boundary, and $Y=\sqrt{\mu / \varepsilon}$ is the surface admittance, the nonphysical reflections will be further diminished [17]. Here, we have introduced $\widetilde{\nabla}=\left(\mathcal{L}_{2, x} \partial / \partial x, \mathcal{L}_{2, y} \partial / \partial y\right)$ to abbreviate the 
notation. Propagation of plane waves with direction $\boldsymbol{\rho}$ is generated in $\Omega_{S}$ by an incident field $H_{z}^{\text {inc }}(\mathbf{r}, t)$ that is introduced on $\Gamma_{\text {inc }} \subset \partial \Omega_{S}$ as

$$
\mathbf{n} \cdot\left(\varepsilon^{-1} \tilde{\nabla} H_{z}\right)-Y(\mathbf{n} \cdot \boldsymbol{\rho}-1) H_{0, z}^{\text {inc }} \frac{\partial f}{\partial t}=0 \quad \text { on } \Gamma_{\text {inc }},
$$

where $H_{0, z}^{\text {inc }}$ and $f(t)$, respectively, are the amplitude and the time evolution of the incident field. When the waves originate from a radiating point source in $\Omega_{S}$, the magnetic charge current becomes $J_{B, z}(\mathbf{r}, t)=\delta\left(\mathbf{r}-\mathbf{r}^{\prime}\right) g(t)$, where $\delta(\mathbf{r})$ denotes the Dirac delta function, $\mathbf{r}^{\prime}$ is the location of the point source, and $g(t)$ is the temporal evolution of the source.

\section{A. Finite-Element Implementation}

To seek the FETD solution of Eq. (1), we employ Galerkin's method [17]. By introducing an appropriate testing function $T_{z}(\mathbf{r})$, the weak-form representation becomes

$$
\begin{gathered}
\iiint_{V}\left[\mu T_{z} \mathcal{L}_{1}(t) H_{z}(t)+\nabla T_{z} \cdot \widetilde{\nabla} H_{z}(t)+T_{z} \frac{\partial J_{B, z}}{\partial t}\right] \mathrm{d} V \\
\quad+\iint_{S}\left[Y_{c} T_{z} \frac{\partial H_{z}}{\partial t}+T_{z} U_{z}\right] \mathrm{d} S=0,
\end{gathered}
$$

where $U_{z}=Y_{c}(\mathbf{n} \cdot \boldsymbol{\rho}-1) H_{0, z}^{\text {inc }} \partial f / \partial t$. Then, expanding the field as

$$
H_{z}(\mathbf{r}, t)=\sum_{i=1}^{N} N_{i}(\mathbf{r}) u_{i}(t),
$$

where $N_{i}$ is the $i$ th interpolating shape function, and assuming that $\mu, \varepsilon, \sigma_{x}$, and $\sigma_{y}$ are constant within each element yields the corresponding finite-element discretization as

$$
\sum_{e=1}^{M}\left(\mathbf{T}^{e} \ddot{\mathbf{u}}+\mathbf{R}^{e} \dot{\mathbf{u}}+\mathbf{S}^{e} \mathbf{u}+\mathbf{g}^{e}-\mathbf{f}^{e}\right)=0,
$$

where $N$ and $M$ denote the numbers of nodes and elements, respectively; $\left(\dot{)} \equiv \mathrm{d} / \mathrm{d} t\right.$; and $(\ddot{)}) \equiv \mathrm{d}^{2} / \mathrm{d} t^{2}$. The square matrices $\mathbf{T}^{e}, \mathbf{R}^{e}$, and $\mathbf{S}^{e}$ are computed by

$$
\begin{gathered}
T_{i j}^{e}=\mu\left\langle N_{i}, N_{j}\right\rangle_{\Omega^{e}}, \\
R_{i j}^{e}=\mu\left(\sigma_{x}+\sigma_{y}\right) \varepsilon_{i}^{-1}\left\langle N_{i}, N_{j}\right\rangle_{\Omega_{\mathrm{PML}}^{e}}+Y_{c}\left\langle N_{i}, N_{j}\right\rangle_{\Gamma_{\mathrm{ABS}}^{e}}, \\
S_{p, i j}^{e}=\varepsilon^{-1}\left\langle\partial N_{i} / \partial p, \partial N_{j} / \partial p\right\rangle_{\Omega^{e}}, \quad p=x, y, \\
S_{i j}^{e}=\mu \sigma_{x} \sigma_{y} \varepsilon_{i}^{-2}\left\langle N_{i}, N_{j}\right\rangle_{\Omega_{\mathrm{PML}}^{e}}+S_{x, i j}^{e}+S_{y, i j}^{e},
\end{gathered}
$$

where $\langle,\rangle_{\Omega^{e}}$ and $\langle,\rangle_{\Gamma^{e}}$ mean integration over the volume and surface, respectively, of an element, and $\mathbf{u}$ $=\left(u_{1}, \ldots, u_{N}\right)^{T}$. Since we model only the scalar field $H_{z}$ it is adequate to consider $N_{i}$ as the nodal based shape function of an element in order to satisfy the field continuity equations. The convolution and the excitation vectors, $\mathbf{g}^{e}$ and $\mathbf{f}^{e}$, respectively, are given by individual vector components:

$$
g_{i}^{e}=\sum_{j} S_{x, i j}^{e} \psi_{x, j}+S_{y, i j}^{e} \psi_{y, j}, \quad e \in \Omega_{\mathrm{PML}}
$$

$$
f_{i}^{e}=-\left\langle N_{i}, \partial J_{B, z} / \partial t\right\rangle_{\Omega_{S}}+\left\langle N_{i}, U_{z}\right\rangle_{\Gamma_{\mathrm{inc}}^{e}}
$$

In Eq. (11) the elements of $\psi_{p}$ are expressed by

$$
\psi_{p, j}=a_{p} \exp \left[-b_{p} t\right] \bar{u}(t) * u_{j}(t), \quad p=x, y .
$$

In Subsection 2.B it is described how the computationally cumbersome convolution term in Eq. (12) is resolved very efficiently to reduce the computational costs.

The $\mathrm{PhC}$ in Fig. 1 is reproduced by using building blocks of size $a / 2 \times \sqrt{3} a / 2$, where $a$ is the basic step length of the $\mathrm{PhC}$, i.e., the lattice constant. These building blocks are discretized in $7 \times 12$ elements yielding 14 elements per inter-hole spacing in the computational mesh, which corresponds to 14 elements per wavelength in the dielectric material.

\section{B. Time Integration}

As a means to speed up the FETD solution in the iterative topology optimization process, we benefit from explicit integration schemes, because they do not require the solution of a matrix system within each time step. Additionally, they have a natural parallelizability. Here, we use a technique that renders the $\mathbf{T}$-matrix diagonal when inversion is needed, and in the case of multiplication an averaged $\mathbf{T}_{\delta}$-matrix is used. The integration of the $\mathbf{T}$ - and S-matrices for a four-node rectangular bilinear element follows modified rules that can be found in [28], such that fourth-order dispersion error accuracy is achieved. A resolution of 14 elements per wavelength is found to be sufficient to obtain acceptable dispersion error. The semidiscrete version of Eq. (9) is given by

$$
\begin{gathered}
\mathbf{T}_{d} \dot{\mathbf{u}}^{n+1 / 2}=\mathbf{T}_{\delta} \mathbf{v}^{n-1 / 2}, \\
\mathbf{T}_{d} \dot{\mathbf{v}}^{n}=-\mathbf{R} \dot{\mathbf{u}}^{n+1 / 2}-\mathbf{S u}^{n}-\mathbf{g}^{n}+\mathbf{f}^{n},
\end{gathered}
$$

where $T_{d, i i}=\Sigma_{j} T_{i j}, T_{d, i j}=0$, and $\mathbf{T}_{\delta}=(1-\delta) \mathbf{T}_{d}+\delta \mathbf{T}$. The optimal combination factor is $\delta=\left(\tau^{2}-1\right) / 2$ to obtain fourthorder accuracy, where $\tau=\Delta t_{c} c / \Delta x$, with $\Delta t_{c}$ denoting the critical time step. It follows the Courant-FriedrichsLewy (CFL)-condition, i.e., $\Delta t_{c} \leq 0.7071 \Delta x / c$, where $c$ is the vacuum speed of light. However, we choose $\Delta t$ $=0.9 \Delta t_{c}$ to avoid or delay numerical instabilities, such as nonphysical oscillations and late-time instabilities in the PML. Half-step approximation is used for central time differences in this scheme, i.e., $\dot{\mathbf{u}}^{n+1 / 2}=\left(\mathbf{u}^{n}-\mathbf{u}^{n-1}\right) / \Delta t$ and $\dot{\mathbf{v}}^{n}=\left(\mathbf{v}^{n+1 / 2}-\mathbf{v}^{n-1 / 2}\right) / \Delta t$, and it is initiated by $\mathbf{u}^{0}=\dot{\mathbf{u}}^{0}=0$ and $\mathbf{v}^{1 / 2}=\Delta t \mathbf{T}_{d}^{-1}\left(\mathbf{f}^{0}-\mathbf{S} \mathbf{u}^{0}\right) / 2$. The temporal derivatives (e.g., of the primal response $\mathbf{u}$ or of the analytical excitation) are evaluated by following the central second difference scheme approximations:

$$
\begin{gathered}
\ddot{\mathbf{u}}^{n}=\frac{\mathbf{u}^{n+1}-2 \mathbf{u}^{n}+\mathbf{u}^{n-1}}{\Delta t^{2}}, \\
\dot{\mathbf{u}}^{n}=\frac{\mathbf{u}^{n+1}-\mathbf{u}^{n-1}}{2 \Delta t} .
\end{gathered}
$$

The convolution term in Eq. (12) requires significant computation time and memory since the entire solution history is needed in the convolution integral. However, it can be recursively evaluated at time $n \Delta t$ instead as [17] 


$$
\psi_{p, j}^{n}=\exp \left[-b_{p} \Delta t\right] \psi_{p, j}^{n-1}+\frac{a_{p} \Delta t}{2}\left(u_{j}^{n}+\exp \left[-b_{p} \Delta t\right] u_{j}^{n-1}\right)
$$

Since $a_{p}$ and $b_{p}$ are assumed to be constant within each element the recursive convolution relation in Eq. (15) is most easily implemented when the matrix-vector multiplication is carried out element-wise in the timemarching. In practice this means that we assign $\psi_{p}^{n}$ separately to each element and update this according to Eq. (15).

\section{Design Variables and Material Interpolation}

In the design domain $\Omega_{D} \subseteq \Omega_{S}$ each finite element is associated with one (density) design variable $x_{e}$ that varies continuously between $0 \leq x_{e} \leq 1$. All design variables are assembled into the global design vector $\mathbf{x}=\left(x_{1}, \ldots, x_{M}\right)^{T}$. By following the solid isotropic material with penalization (SIMP) scheme [23] the design variable is then used to interpolate between two candidate material phases, designated here as ( $)^{I}$ for air and ( $)^{I I}$ for dielectric. Since relative magnetic permeability is very close to unity for dielectric materials, explicit design dependence is only restricted to the inverse of the relative permittivity. This is successfully adopted by a linear interpolation [6],

$$
\varepsilon_{r}^{-1}\left(x_{e}\right)=\left(1-x_{e}\right)\left(\varepsilon_{r}^{I}\right)^{-1}+x_{e}\left(\varepsilon_{r}^{I I}\right)^{-1} .
$$

The continuous design parameterization above now facilitates the use of a gradient-based optimization algorithm (referred to as the optimizer) to find an optimized design. The shortcoming of the continuous material parameterization is the possible scenario of intermediate design variables appearing in the final design. In Subsection 2.E it is described how design variables close to discrete $\left(x_{e}\right.$ $=0$ and $x_{e}=1$ ) are efficiently obtained through penalization schemes.

\section{Sensitivity Analysis}

In our continuous optimization problem we need to compute the design sensitivities in order to use gradientbased optimization solvers. For large numbers of design variables the adjoint sensitivity approach offers a clever and computationally efficient alternative to direct sensitivity analysis [29]. As briefly described, the adjoint method makes the central processing unit time associated with the sensitivity analysis almost independent on the number of design variables by introducing an auxiliary problem (the adjoint problem) that needs to be solved backward in time.

The development of an explicit design sensitivity expression proceeds as follows [30]. Consider a design functional $\Phi$ that is defined here as a function of the design $\mathbf{x}$ :

$$
\Phi(\mathbf{x})=\int_{0}^{T} F(\mathbf{u}, \dot{\mathbf{u}}, \ddot{\mathbf{u}}, \mathbf{x}) \mathrm{d} t,
$$

where $T$ is the termination time of the transient simulation, and $\mathbf{u} \equiv \mathbf{u}(\mathbf{x}, t)$ where the design dependence is implicit. Now, we continue by expressing the sensitivities in terms of the residual of Eq. (9), given by

$$
\mathbf{r}(\mathbf{u}, \dot{\mathbf{u}}, \ddot{\mathbf{u}}, \mathbf{x})=\mathbf{f}-(\mathbf{T} \ddot{\mathbf{u}}+\mathbf{R} \dot{\mathbf{u}}+\mathbf{S u}+\mathbf{g})=\mathbf{0} .
$$

Combining the integrand in Eqs. (17) and (18) defines the augmented functional

$$
\hat{F}=F(\mathbf{u}, \dot{\mathbf{u}}, \ddot{\mathbf{u}}, \mathbf{x})+\lambda^{T} \mathbf{r}(\mathbf{u}, \dot{\mathbf{u}}, \ddot{\mathbf{u}}, \mathbf{x})
$$

where the adjoint operator (Lagrange multiplier) $\boldsymbol{\lambda}$ $\equiv \boldsymbol{\lambda}(\mathbf{x}, t)$ depends implicitly on the design and explicitly on time. The augmented design functional is identical to that in Eq. (17) with $F$ replaced with $\hat{F}$, though, since $\mathbf{r}=\mathbf{0}$. By a clever choice of $\boldsymbol{\lambda}$, which can be chosen freely since $\mathbf{r}$ $=\mathbf{0}$, the sensitivity analysis is significantly simplified as shown in the following.

Now, differentiating Eq. (17) with respect to each component $x_{e} \in \mathbf{x}$ by the chain rule yields the sensitivities

$$
\begin{aligned}
\frac{\partial \Phi}{\partial x_{e}}= & \int_{0}^{T}\left(\frac{\partial F}{\partial \mathbf{u}} \frac{\partial \mathbf{u}}{\partial x_{e}}+\frac{\partial F}{\partial \dot{\mathbf{u}}} \frac{\partial \dot{\mathbf{u}}}{\partial x_{e}}+\frac{\partial F}{\partial \ddot{\mathbf{u}}} \frac{\partial \ddot{\mathbf{u}}}{\partial x_{e}}+\frac{\partial F}{\partial x_{e}}+\frac{\partial \boldsymbol{\lambda}^{T}}{\partial x_{e}} \mathbf{r}\right. \\
& \left.+\boldsymbol{\lambda}^{T}\left[\frac{\partial \mathbf{r}}{\partial \mathbf{u}} \frac{\partial \mathbf{u}}{\partial x_{e}}+\frac{\partial \mathbf{r}}{\partial \dot{\mathbf{u}}} \frac{\partial \dot{\mathbf{u}}}{\partial x_{e}}+\frac{\partial \mathbf{r}}{\partial \mathbf{u}} \frac{\partial \ddot{\mathbf{u}}}{\partial x_{e}}+\frac{\partial \mathbf{r}}{\partial x_{e}}\right]\right) \mathrm{d} t .
\end{aligned}
$$

Utilizing integration by parts and that $\mathbf{r}=\mathbf{0}$, Eq. (20) is now rewritten as

$$
\begin{aligned}
\frac{\partial \Phi}{\partial x_{e}}= & {\left[\boldsymbol{\lambda}^{T} \frac{\partial \mathbf{r}}{\partial \dot{\mathbf{u}}} \frac{\partial \mathbf{u}}{x_{e}}+\boldsymbol{\lambda}^{T} \frac{\partial \mathbf{r}}{\partial \ddot{\mathbf{u}}} \frac{\partial \dot{\mathbf{u}}}{x_{e}}-\dot{\boldsymbol{\lambda}}^{T} \frac{\partial \mathbf{r}}{\partial \ddot{\mathbf{u}}} \frac{\partial \mathbf{u}}{\partial x_{e}}\right]_{0}^{T} } \\
& +\int_{0}^{T}\left(\frac{\partial F}{\partial \dot{\mathbf{u}}} \frac{\partial \dot{\mathbf{u}}}{\partial x_{e}}+\frac{\partial F}{\partial \ddot{\mathbf{u}}} \frac{\partial \ddot{\mathbf{u}}}{\partial x_{e}}+\frac{\partial F}{\partial x_{e}}\right) \mathrm{d} t \\
& +\int_{0}^{T}\left(\frac{\partial \mathbf{r}}{\partial \ddot{\mathbf{\lambda}}}-\frac{\partial \mathbf{r}}{\partial \dot{\mathbf{u}}} \dot{\boldsymbol{\lambda}}+\frac{\partial \mathbf{r}}{\partial \mathbf{u}} \boldsymbol{\lambda}-\frac{\partial F}{\partial \mathbf{u}}\right) \frac{\partial \mathbf{u}}{\partial x_{e}} \mathrm{~d} t+\int_{0}^{T} \boldsymbol{\lambda}^{T} \frac{\partial \mathbf{r}}{\partial x_{e}} \mathrm{~d} t .
\end{aligned}
$$

The implicit system derivatives $\partial \mathbf{u} / \partial x_{e}$ are eliminated from the sensitivity expression by selecting the appropriate $\boldsymbol{\lambda}$. This process induces the adjoint problem

$$
\frac{\partial \mathbf{r}}{\partial \ddot{\mathbf{u}}} \ddot{\boldsymbol{\lambda}}-\frac{\partial \mathbf{r}}{\partial \dot{\mathbf{u}}} \dot{\boldsymbol{\lambda}}+\frac{\partial \mathbf{r}}{\partial \mathbf{u}} \boldsymbol{\lambda}=\frac{\partial F}{\partial \mathbf{u}},
$$

where $\partial F / \partial \mathbf{u}$ designates the adjoint load. If we introduce the time shift $t \equiv T-\tau$ for $\tau \in[0, T]$, the adjoint problem in terms of $\boldsymbol{\lambda}(\tau)$ becomes

$$
\frac{\partial \mathbf{r}}{\partial \ddot{\mathbf{u}}}+\frac{\partial \mathbf{r}}{\partial \dot{\mathbf{u}}} \dot{\overline{\mathbf{u}}}+\frac{\partial \mathbf{r}}{\partial \mathbf{u}} \overline{\boldsymbol{\lambda}}=\frac{\partial F}{\partial \mathbf{u}},
$$

since $\partial / \partial t=-\partial / \partial \tau$. Then upon imposing $\mathbf{u}(0)=\dot{\mathbf{u}}(0)=\mathbf{0}$, solving Eq. (23) with the terminal condition $\overline{\boldsymbol{\lambda}}(0)=\dot{\bar{\lambda}}(0)$ $=\mathbf{0}$, and subsequently substituting $\boldsymbol{\lambda}(T-\tau)=\overline{\boldsymbol{\lambda}}(\tau)$, the sensitivity expression reduces to

$$
\frac{\partial \Phi}{\partial x_{e}}=\int_{0}^{T}\left(\frac{\partial F}{\partial x_{e}}+\lambda^{T} \frac{\partial \mathbf{r}}{\partial x_{e}}\right) \mathrm{d} t .
$$

In our case $\partial F / \partial \dot{\mathbf{u}}$ and $\partial F / \partial \ddot{\mathbf{u}}$ vanish since we only consider problems in which $F=F(\mathbf{u}, \mathbf{x})$. The result is an adjoint problem whose form is identical to that of the primal transient analysis in Eq. (9), albeit with another excita- 
tion term. Hence, the same time integration scheme can be used to find the adjoint response.

The evaluation of the adjoint sensitivities proceeds as follows: After the transient primal analysis is concluded, the adjoint response $\boldsymbol{\lambda}$ is computed at the exact same time steps by reusing the exact same ABC setup from the primal analysis [30]. While integrating the adjoint response in time, the contribution to the integral in Eq. (24) is computed, which is plausible since $\boldsymbol{\lambda}$ and $\mathbf{u}$ are already known. Consequently, when integration of the adjoint response is concluded the sensitivities are obtained. This process only requires the storage of the primal response $\mathbf{u}(t)$ from which, when needed, $\ddot{\mathbf{u}}(t)$ and $\dot{\mathbf{u}}(t)$ are computed by Eq. (14).

More often, it is desirable to control the objective in a localized time interval $\left[T_{1}, T\right] \subseteq[0, T]$ (see [31]). The lower integration limit in Eq. (17) cannot simply be replaced with $T_{1}$, because then the brackets in Eq. (21) will not vanish. Alternatively, the time integral in Eq. (17) could be split into two integrals $\int_{T_{1}}^{T}=\int_{0}^{T}-\int_{0}^{T_{1}}$. However, this requires two adjoint analyses. In order to reduce the computational costs we suggest incorporating a localizing function in the design functional instead. The Heaviside step size function $\bar{u}\left(t-T_{1}\right)$ allows us to specify a time interval, such that the design functional becomes

$$
\Phi(\mathbf{x})=\int_{0}^{T} F(\mathbf{u}, \dot{\mathbf{u}}, \ddot{\mathbf{u}}, \mathbf{x}) \bar{u}\left(t-T_{1}\right) \mathrm{d} t,
$$

which modifies the adjoint load to become $[\partial F / \partial \mathbf{u}] \bar{u}(t$ $\left.-T_{1}\right)$.

\section{E. Optimization Problem}

In the present optimization formulation, the design functional $\Phi(\mathbf{x})$ is restricted to scalar measures. Here, we consider magnetic energy given by $\int_{\Omega_{E}} \frac{1}{2} \mu H_{z}^{2} \mathrm{~d} \Omega$. Thus, $F$ can in general be expressed as $\mathbf{u}^{T} \mathbf{Q u}$, where $\mathbf{Q}$ is a matrix with the components $Q_{i j}^{e}=\frac{1}{2} \mu\left\langle N_{i}, N_{j}\right\rangle_{\Omega^{e}}$ for $e \in \Omega_{E}$. In PhC-MC design problems the $Q$ factor can be enhanced by delaying the exponential energy decay [32]. To achieve this, we suggest including the localizing function in $F$ to specify the elapsed time, after which the magnetic energy should be maximized. The optimization problem is now formulated as

$$
\begin{gathered}
\max _{\mathbf{x} \in \mathbf{R}^{M}} \Phi(\mathbf{x})=\log _{10}\left[\int_{0}^{T} \mathbf{u}^{T} \mathbf{Q} \mathbf{u} \bar{u}\left(t-T_{1}\right) \mathrm{d} t\right], \\
\text { s.t.: Governing Eq. (9) } \\
\sum_{e} v_{e} x_{e}<V f^{*}, \quad 0 \leq x_{e} \leq 1, \quad e \in \Omega_{D} .
\end{gathered}
$$

In the second constraint $v_{e}$ is the element volume, and $V$ is the total volume occupied by $\Omega_{D}$; hence $V=\Sigma_{e} v_{e}$. The constraint serves as a restriction on the available amount of dielectric material, set by $f^{*}$ herein, that is to be distributed in $\Omega_{D}$. It is important to stress that it is not necessarily active in this problem. The logarithm in the objective is introduced to ensure better numerical scaling. The optimization process is initiated by a qualified design that is found via the trial and error approach or previously reported designs.

The solution of the optimization problem in Eq. (26) suffers from strong non-uniqueness leading to multiple local minima. Some of them are a result of the possible existence of degenerate modes [3]. Others stem from local resonance effects yielding a poor performance away from the target frequency [7]. Since the physics behind sharp resonance phenomena induces very sensitive behavior upon tiny design changes, we use a globally convergent optimizer. Thereby we stay in the vicinity of the initial design and avoid undesirable minima. Based on an initial design and the adjoint sensitivities, the design update is carried out by the globally convergent method of moving asymptotes that is suggested and implemented in Fortran 77 by Svanberg [33]. The Heaviside step function $\bar{u}(t$ $-T_{1}$ ) in Eq. (26) is regularized in a neighborhood $\Delta T$ centered at $T_{1}$ by

$$
\bar{u}\left(t-T_{1}\right) \approx \frac{1}{2} \frac{\tanh \left[2 \beta\left(t-T_{1}\right) / \delta T\right]}{\tanh [\beta]}+\frac{1}{2}
$$

to avoid numerical problems. The parameter $\beta$ dictating the curvature of the regularization and the size of $\delta T$ are chosen such that the fast Fourier transform of $\mathbf{u}^{T} \mathbf{Q u} \bar{u}(t$ $-T_{1}$ ) does not disclose any undesirable local resonance peaks away from the target frequency of the mode.

In order to avoid any non-manufacturable features in the structure we use density filtering techniques capable of controlling the minimum length scale of void and dielectric simultaneously [34,35]. However, this particular type of multiphase projection leads to intermediate design variables in the transition region between the material phases. We use the so-called pamping method [7] that introduces an artificial mass proportional damping contribution $\mathbf{R}^{e}=4 q x_{e}\left(1-x_{e}\right) \omega_{0} \mathbf{T}^{e}$ and makes the existence of intermediate design variables expensive with respect to the objective. This penalization procedure is, however, only applicable in this form for maximization problems. Numerical experiments have proven $q=0.2\left(T-T_{1}\right) / T$ to be an adequate choice.

The state problem solver, providing the objective and sensitivity evaluation, is parallellized by using Fortran 90 MPI for all interprocessor communications to avoid computational bottlenecks in the storage of the primal response. A non-blocking communication strategy is implemented in the time integration to speed up the simulation time. The design update is carried out on a single processor, to which all necessary data for the optimizer are sent.

\section{DESIGN OF A SIDE-COUPLED RESONANT CAVITY}

The high index-contrast devices used in the present investigation are constructed by using a $2 \mathrm{D} \mathrm{PhC}$ composed of a triangular lattice of air holes embedded in the dielectric material GaAs (see Fig. 1). By viewing the structures as infinite in the third dimension and using the radius of the air holes $r / a=0.35$, it supports a complete $\mathrm{TE}_{z}$ bandgap in the normalized frequency range $a / \lambda_{0}=0.21-0.33$, where 
$\lambda_{0}$ denotes the wavelength in vacuum. The dielectric contrast between GaAs and air is 11.4 for wavelengths around $1.5 \mu \mathrm{m}$ [3].

The side-coupled cavity in the $2 \mathrm{D} \mathrm{PhC}$ with an infinite height has two distinct loss mechanisms. One concerns the leak of the cavity mode into the $\Gamma-K$ directional WG, and the other concerns that into the surrounding $\mathrm{PhC}$. Hence, the total in-plane $Q$ factor is given by

$$
1 / Q_{\text {in }}=1 / Q_{e}+1 / Q_{0},
$$

where $Q_{e}$ is the quality factor of the cavity mode with respect to the WG, and $Q_{0}$ is the quality factor of the isolated cavity. From Eq. (28) it is deduced that $Q_{\text {in }}$ increases when the cavity is far away from the WG, albeit at the expense of poor coupling. In fact, $Q_{\text {in }}$ increases exponentially with the distance [20]. Since the ultimate goal is miniaturized integrated $\mathrm{PhC}$ circuit devices, the method of topology optimization is utilized here to improve $Q_{\text {in }}$ for short distances while maintaining strong coupling. The first target in the design process is to improve $Q_{0}$ by considering the isolated cavity.

\section{A. Isolated Cavity Design}

Although an arbitrary initial condition could be employed, it is reasonable to search for a cavity geometry supporting a high $Q$ mode by varying the radii of nearest neighboring holes to the cavity. The geometry in Fig. 2(a) that is surrounded by a two cell sizes thick PML pulls down a monopole $H_{z}$-mode from the air band at the fre-

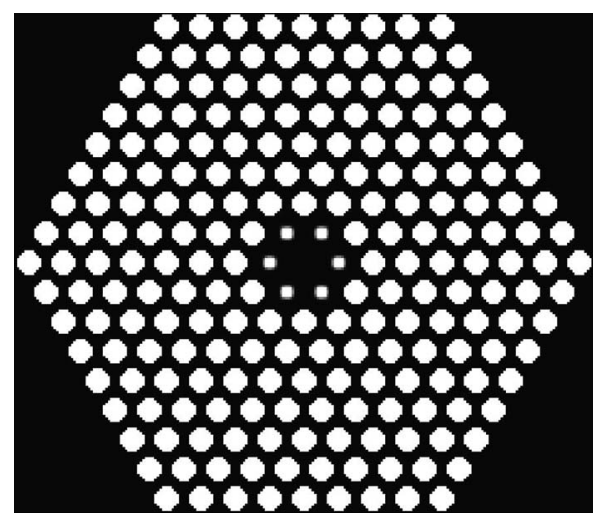

(a)

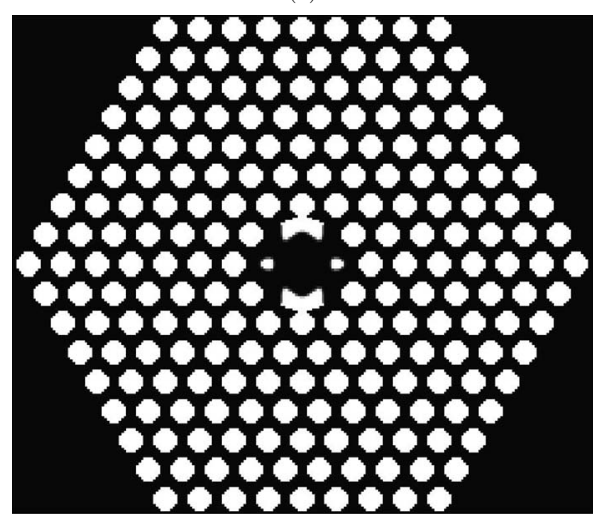

(b)

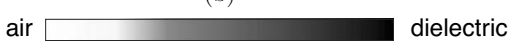

Fig. 2. Monopole mode. (a) Initial MC geometry. (b) Optimized MC geometry. quency of $\omega_{0} a / 2 \pi c=0.3030$ with $Q_{0}=2.2 \times 10^{5}$. We calculate the $Q$ factor by measuring the slope of the exponential decay of the energy of the cavity mode [32]

$$
U(t)=U_{0} \exp \left[-\omega_{0} t / Q_{0}\right]
$$

where $U_{0}$ represents the (initial) amount of energy stored in the cavity that is reached at time $T_{\max }$. The corresponding mode profile in Fig. 3(b) is extracted by the discrete Fourier transform of the response that is excited by a radiation point source in the center of the cavity with time evolution

$$
g(t)=\exp \left[-\left(t-T_{0}\right)^{2} / \tau^{2}\right] \sin \left[2 \pi \omega_{0}\left(t-T_{0}\right)\right]
$$

where $\tau=180 \mathrm{c} / \mathrm{a}$ and $T_{0}=500 \mathrm{a} / \mathrm{c}$.

The design region $\Omega_{D}$ for the coupled system depicted in Fig. 1 is also adopted for isolated MC optimization. In the center hereof the objective is evaluated in a circular region $\Omega_{E}$ with radius $3 a / 14$. We excite the system by reusing the time evolution in Eq. (30), and the simulation time is set to $T=100,000 \Delta t$. The choice of $T_{1}$ is not critical here since the isolated cavity has only a single loss mechanism. Additionally, we impose vertical and horizontal symmetry conditions through the center of the cavity and do not constrain the amount of the dielectric material, i.e., $f^{*}=1$. The optimized design reached after 311 design iterations appears in Fig. 2(b). As expected it does not include any structural features that violate the minimum allowable length scale prescribed by the density filter with radius $2.5 a / 14$, and the length scale of the blending region between dielectric and air corresponds to the thickness of one finite element. Based on the normalized logarithmic energy decay in Fig. 3(a) the $Q$ factor of the corresponding mode is computed to be $Q_{0}=4.8 \times 10^{5}$. Evidently, the initial design has been altered significantly to achieve this doubling of the $Q$ factor, likewise the mode profile in Fig. 3(c).

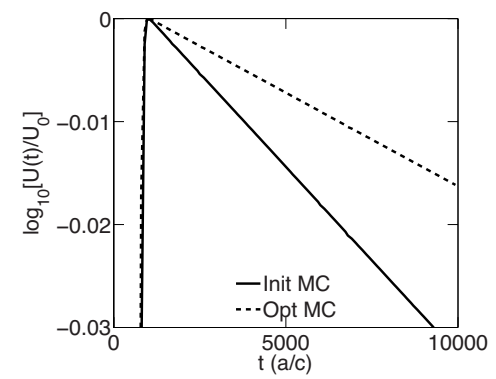

(a)

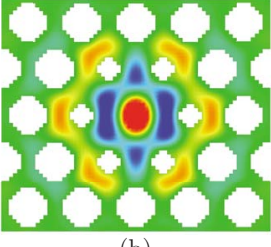

(b)

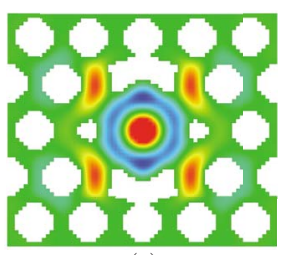
pos
Fig. 3. (Color online) (a) Logarithmic envelope of normalized stored energy for the monopole mode. (b), (c) $H_{z}$-field distribution for the initial and optimized MC geometries, respectively. The material distribution is shown with $x_{e}^{t}=0.6$ as threshold. 


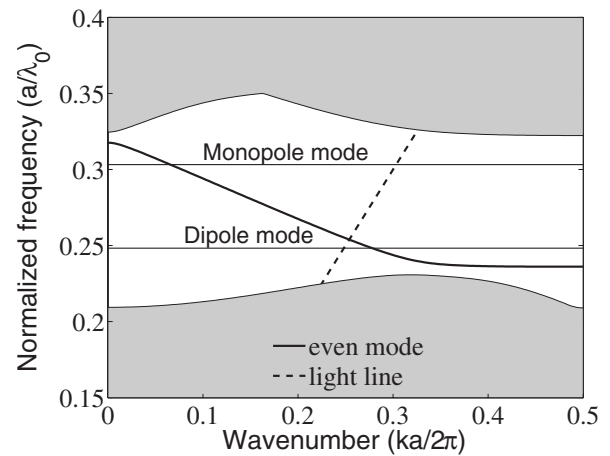

Fig. 4. Dispersion curve of the $\Gamma-K$ directional $\mathrm{PhC}-\mathrm{WG}$. The bandgap exists between the dielectric (lower) and air (higher) bands in the normalized frequency range $a / \lambda_{0}=0.21-0.33$. The horizontal lines represent the dipole mode frequency of 0.2480 and the monopole mode frequency of 0.3030 .

In practical applications of the side-coupled MC the initial geometry and corresponding mode cannot be chosen uncritically. The influence of the light line in the dispersion diagram should then be taken into account. Unlike for the monopole mode, we identify a dipole mode at the frequency of $\omega_{0} a / 2 \pi c=0.2480$ whose intersection point with the WG mode is below the light line; see the dispersion diagram in Fig. 4. The corresponding MC geometry appears in Fig. 5(a).

For the dipole mode $\Omega_{D}$ remains unchanged, and the radius of $\Omega_{E}$ is now doubled to $6 a / 14$. The system is excited by two point sources located in the eye of the dipole

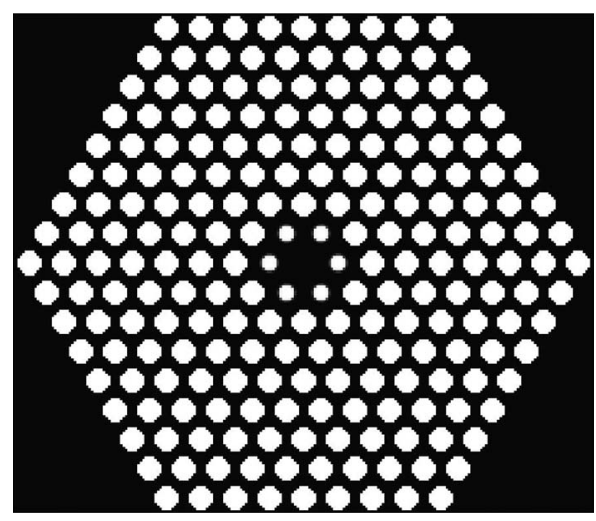

(a)

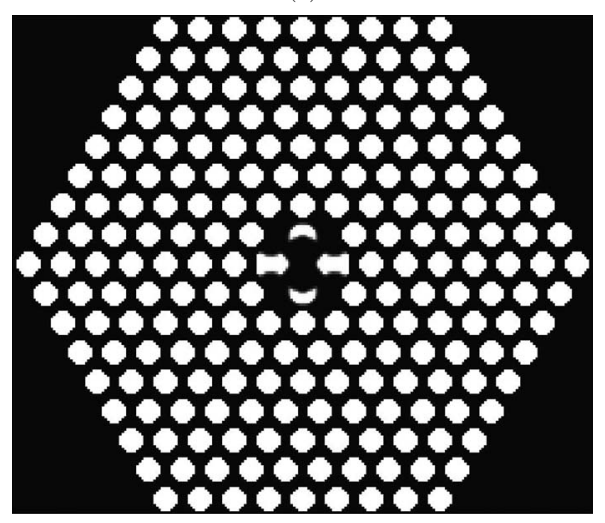

(b)

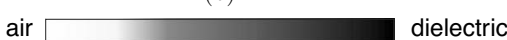

Fig. 5. Dipole mode. (a) Initial MC geometry. (b) Optimized MC geometry. with opposite signs. Now, however, it has been necessary to constrain the amount of the dielectric material to $f^{*}$ $=0.8$ to avoid ending up in local maxima bearing degenerate modes. The optimized geometry reached after 592 design iterations is displayed in Fig. 5(b). Compared to the mode in Fig. 6(b) of the initial design with $Q_{0}=9.9 \times 10^{4}$ the $Q$ factor of the optimized design is slightly improved to $Q_{0}=11 \times 10^{4}$ [see Fig. 6(a)]. Similar to the monopole mode, the topological features of the optimized design prevent the cavity field in Fig. 6(c) from leaking into the surrounding $\mathrm{PhC}$ above and below the cavity. Instead, the leakage is only concentrated on the corners.

\section{B. Coupled System Design}

For the coupled system in Fig. 1 we consider, for the sake of completeness, both cavity modes despite the limited practical applicability of the monopole. Here, we impose only a vertical symmetry condition through the cavity center to increase the design freedom. The system is excited by a line source $\Gamma_{\text {inc }}$ located inside the PhC-WG with an appropriate distance from the cavity and with temporal dependence given by Eq. (30). The entire structure is surrounded by a PML into which the $\mathrm{PhC}$ features are continued (see Fig. 1). The design process is initiated by reusing the optimized isolated cavity designs with similar $\Omega_{D}$ and $\Omega_{E}$, and the simulation time is set to $T$ $=120,000 \Delta t$. Since the coupled system now supports multiple loss mechanism, the choice of $T_{1}$ becomes crucial in order to improve $Q_{\text {in }}$. Here, the three cases $T_{1}=0,=T_{\max }$, and $=4 T_{\max }$ are considered. We compute $Q_{\text {in }}$ by utilizing that $\omega_{0} / Q$ equals the full width at half-maximum (FWHM) of the resonant shape of the transmitted power ratio $|T|^{2} \equiv P_{\text {out }} / P_{\text {in }}$ for the coupled device. Similarly, however, by varying the separation between the PhC-WG and -MC determines $Q_{e}$. The power is computed by the Poynting vector at the flux plane $\Gamma_{\text {out }}$, given by

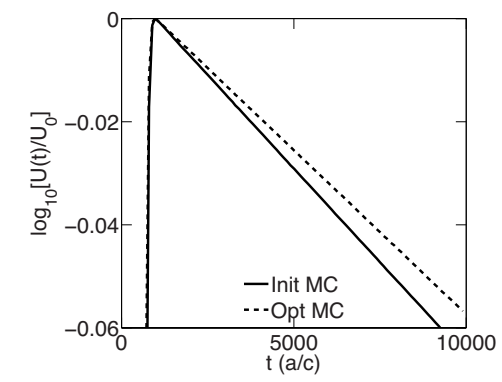

(a)

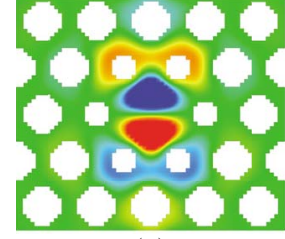

(b)

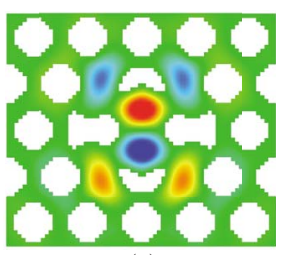

(c) pos
Fig. 6. (Color online) (a) Logarithmic envelope of normalized stored energy for the dipole mode. (b), (c) $H_{z}$-field distribution for the initial and optimized MC geometries, respectively. The material distribution is shown with $x_{e}^{t}=0.6$ as threshold. 


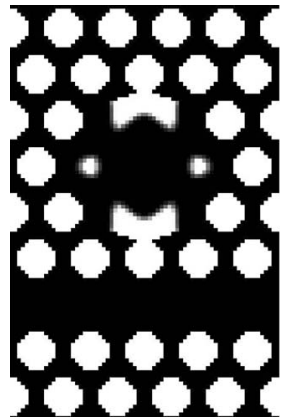

(a)

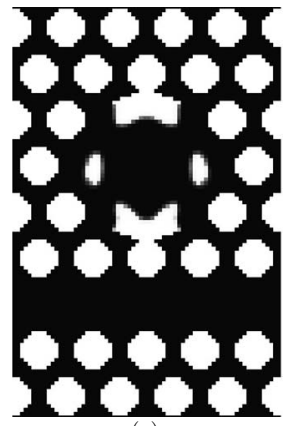

(c)

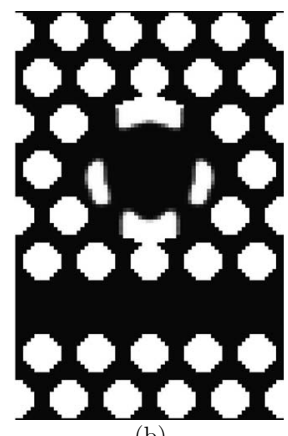

(b)

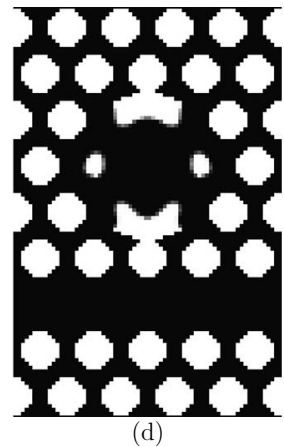

Fig. 7. Optimized designs for the monopole MC mode. (a) Isolated MC. Optimized coupled system geometries for (b) $T_{1}=0$, (c) $T_{1}=T_{\max }$, and (d) $T_{1}=4 T_{\max }$.

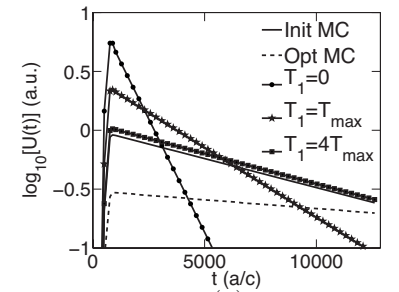

(a)

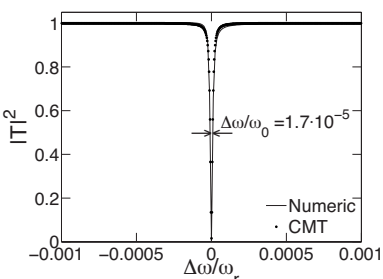

(c)

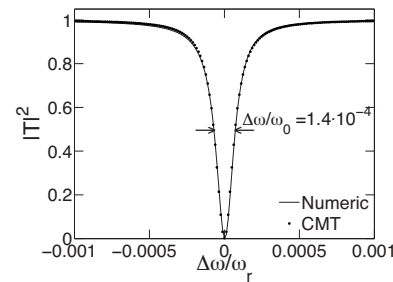

(e)

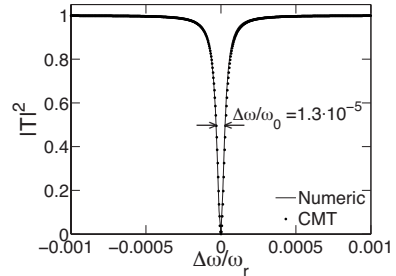

(b)

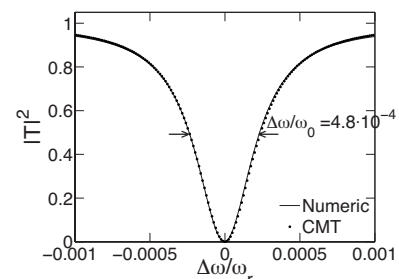

(d)

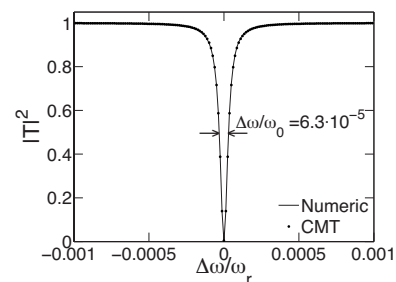

(f)
Fig. 8. Monopole mode. (a) Logarithmic envelope of stored energy $U(t)$. Transmission spectrum for (b) initial design, (c) isolated MC, coupled system (d) $T_{1}=0$, (e) $T_{1}=T_{\max }$, and (f) $T_{1}$ $=4 T_{\max }$.

$$
P(\omega)=\frac{1}{2} \operatorname{Re}\left[\mathbf{n} \cdot \int_{\Gamma_{\text {out }}} \mathbf{E}_{\omega}^{*} \times \mathbf{H}_{\omega} \mathrm{d} \Gamma\right]
$$

where ( )* denotes complex conjugate, and ( ) ${ }_{\omega}$ means discrete Fourier transform of the time dependent field. By removing the scattering cavity $P_{\text {in }}$ can be determined at $\Gamma_{\text {out }}$. For a broadband analysis, the frequency span of the incoming Gaussian wave packet corresponds to that of the guided mode.

The optimized designs for the monopole and dipole modes follow from Figs. 7 and 9, respectively, and none of those contravene the similar minimum allowable length scale as above. For the monopole mode, the results in Figs. 7(b)-7(d) reveal that the optimization has caused minor redistribution of the dielectric material. According to Figs. 8(b)-8(f) the FWHM of the Lorentzian dip in transmission indicates that $Q_{\text {in }}$ is deteriorated compared to the performance of the isolated cavity design. An overview of all relevant $Q$ factors is presented in Table 1 . The significance of choosing $T_{1}$ is illustrated by the envelope of the stored energy response inside the cavity in Fig. 8(a). When $T_{1}$ approaches zero the WG energy attempts to couple into the cavity immediately yielding a strong interaction and a subsequent rapid decay inside the cavity as a consequence of low $Q_{\text {in }}$. As $T_{1}$ grows beyond the $T_{\max }$-limit, the energy decay is postponed, which suggests that achieving high $Q$ devices in principle counteracts strong coupling. Nevertheless, in all cases almost zero transmission is achieved independent of the difference in $Q_{\text {in. }}$. This behavior can be understood by utilizing CM theory [24] to obtain an analytical expression for the transmission in the WG, given by

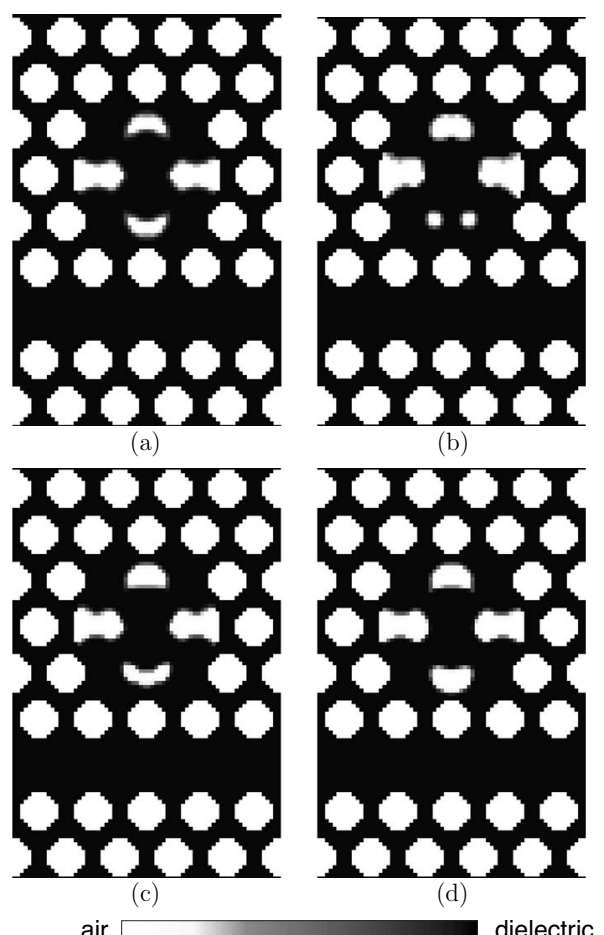

air

dielectric

Fig. 9. Optimized designs for the dipole MC mode. (a) Isolated MC. Optimized coupled system geometries for (b) $T_{1}=0$, (c) $T_{1}$ $=T_{\max }$, and (d) $T_{1}=4 T_{\max }$. 
Table 1. $Q\left[10^{3}\right]$ Factors for Coupled System Configurations

\begin{tabular}{|c|c|c|c|c|c|c|}
\hline & \multicolumn{3}{|c|}{ Monopole } & \multicolumn{3}{|c|}{ Dipole } \\
\hline & $Q_{\text {in }}$ & $Q_{e}$ & $Q_{0}$ & $Q_{\text {in }}$ & $Q_{e}$ & $Q_{0}$ \\
\hline Init. MC & 16 & 17.3 & 220 & 2.1 & 2.1 & 99 \\
\hline Opt. MC & 60 & 68.3 & 474 & 4.1 & 4.2 & 110 \\
\hline$T_{1}=0$ & 2.1 & 2.1 & 139 & 2.0 & 2.0 & 90 \\
\hline$T_{1}=T_{\max }$ & 7 & 7.3 & 168 & 3.3 & 3.4 & 100 \\
\hline$T_{1}=4 T_{\max }$ & 16 & 17.2 & 239 & 6.0 & 6.2 & 140 \\
\hline
\end{tabular}

$$
|T(\omega)|^{2}=1-\frac{\frac{1}{4 Q_{e}^{2}}\left(1+2 \frac{Q_{e}}{Q_{0}}\right)}{\left(\frac{\omega-\omega_{0}}{\omega_{0}}\right)^{2}+\frac{1}{4 Q_{e}}\left(1+\frac{Q_{e}}{Q_{0}}\right)^{2}} .
$$

At resonance, i.e., $\omega=\omega_{0}$, it simplifies to

$$
\left|T\left(\omega_{0}\right)\right|^{2}=\frac{Q_{\mathrm{in}}^{2}}{Q_{0}^{2}} \approx \frac{Q_{e}^{2}}{Q_{0}^{2}}+O\left(\left[Q_{e} / Q_{0}\right]^{3}\right),
$$

where the last approximation is valid if $Q_{0} \gg Q_{e}$. Table 1 indicates that it holds true for the present system, and additionally that $Q_{e}$ and $Q_{\text {in }}$ have the same order of magnitude. Hence at resonance according to Eq. (33), strong coupling is retained even upon considerable improvement of $Q_{\text {in }}$ (and $Q_{e}$ ). Consequently, the optimization formulation makes it possible to control the counteracting relation between high $Q$ and strong coupling. One should also expect that $Q_{0}$ is largest for the isolated cavity optimization. Table 1 supports this fact for the monopole. How-

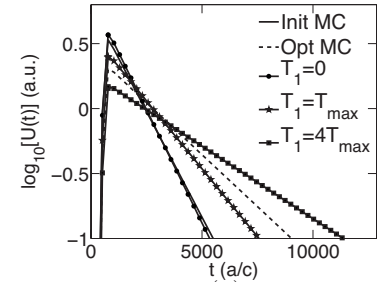

(a)

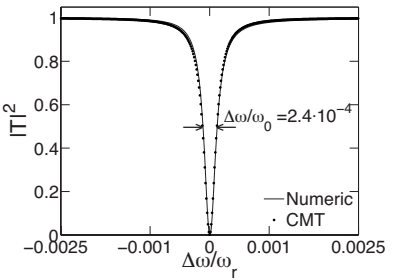

(c)

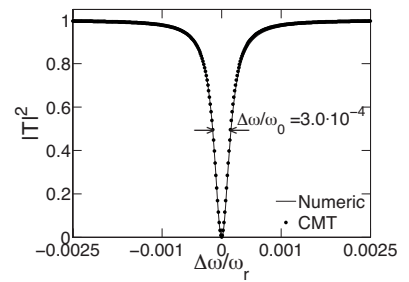

(e)

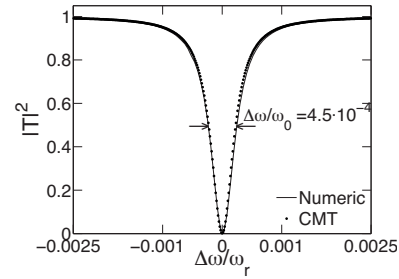

(b)

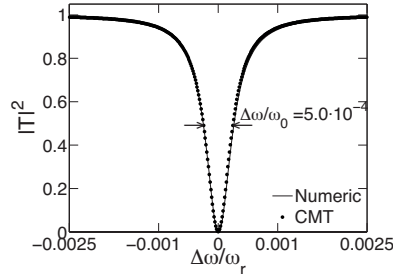

(d) ${ }^{r}$

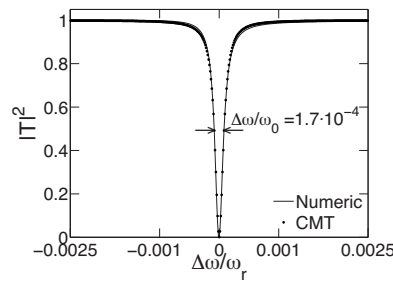

(f)
Fig. 10. Dipole mode. (a) Logarithmic envelope of stored energy $U(t)$. Transmission spectrum for (b) initial design, (c) isolated MC, coupled system (d) $T_{1}=0$, (e) $T_{1}=T_{\max }$, and (f) $T_{1}=4 T_{\text {max }}$. ever, the dipole mode favors the largest $Q_{0}$ for unsymmetrical cavity design in the vertical direction. This is only obtainable for the coupled system $\left(T_{1}=4 T_{\max }\right)$ as a result of the symmetry conditions in both directions for the isolated cavity optimization.

For the dipole mode the original cavity geometry in Fig. 5(a) and the optimized ones in Fig. 9 exhibit practically zero transmission at resonance [see Figs. 10(b)-10(f)]. The isolated cavity optimization only improves $Q_{\text {in }}$ by a factor of 2 . In contrast to the monopole, optimizing for $T_{1}=4 T_{\text {max }}$ further increases $Q_{\text {in }}$ by $50 \%$ as a result of minor design changes [compare Figs. 9(a) and 9(d)], yielding a total improvement of $185 \%$. The design result for $T_{1}$ $=0$ in Fig. 5(a) shows substantial material redistribution in the interaction region in order to enhance coupling. However, the envelopes of the energy decay in Fig. 10(a) only display marginal changes among the various designs in the coupling performance. As expected the $H_{z}$-field plot in Fig. 11 of the best dipole candidate $\left(T_{1}=4 T_{\text {max }}\right)$ shows nearly zero transmission at resonance.

The use of a $2 \mathrm{D} \mathrm{PhC}$ of infinite height in this paper omits the influence of the out-of-plane energy losses, which constitutes a limiting factor with regards to improving the total $Q$ factor in $\mathrm{PhC}$ slabs. Thus, improving the in-plane $Q$ does not necessarily imply the same trend for the out-of-plane $Q$ [22]. Furthermore, we cannot take the third dimension into account by the effective index method since it holds limited accuracy for high indexcontrast structures or near the cutoff. However, our experience from previous studies (cf. [9-12]) is that $2 \mathrm{D}$

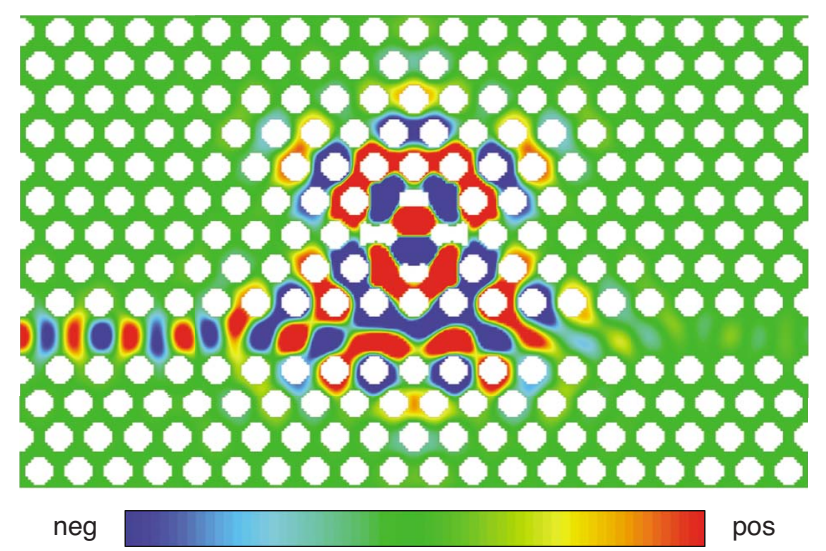

Fig. 11. (Color online) $H_{z}$-field distribution for the best optimized WG-side-coupled MC candidate for the dipole mode when $T_{1}=4 T_{\max }$. The material distribution is shown with $x_{e}^{t}=0.6$ as threshold. 
optimized designs in general yield good behavior in three dimensions as well. In future studies we will verify the obtained designs by three-dimensional simulations and possibly extend the optimization to three dimensions as well.

\section{CONCLUSION}

In this paper, we have developed a design method based on topology optimization for transient response, and we have used it to design a miniaturized PhC-WG-sidecoupled PhC-MC device with an improved $Q$ factor while maintaining strong coupling. Frankly we have shown that the transient optimization formulation makes us capable of controlling the counteracting relation between high $Q$ factor and strong coupling.

The optimization algorithm relies on a 2D FETD model for TE-polarized waves that uses PMLs as absorbing boundary conditions (ABCs), which has not been reported before. To manage the material distribution of air and dielectric we associate a continuously varying design variable to each element in the design domain enclosing the MC. We suggest that the in-plane $Q$ factor can be improved by maximizing the stored cavity energy in the decaying regime of the transient response. Manufacturable designs are achieved by filtering techniques that control the minimum length scale of air and dielectric simultaneously.

The design process is threefold. First, by trial and error we alternate the radii of nearest neighboring holes to the cavity to find a well-performing geometry. This serves as an initial guess in the optimization of the isolated cavity, and if the performance is improved, this is subsequently used to start the optimization of the coupled device. We have optimized the coupling to a monopole and a dipole MC mode, and in both cases we managed to improve the in-plane $Q$ factors by $275 \%$ and $185 \%$, respectively, compared to the original design. To study the spectral performance of the optimized designs we have derived an analytical expression for the transmission based on coupledmode (CM) theory. These agree very well with numerical simulations.

\section{ACKNOWLEDGMENTS}

This work is supported by the Eurohorcs/ESF European Young Investigator Award (EURYI) [36] through the grant "Synthesis and topology optimization of optomechanical systems." The Danish Center for Scientific Computing is gratefully acknowledged. The authors are grateful to members of the TopOpt-group [37] for fruitful discussions related to the presented work.

\section{REFERENCES}

1. S. John, "Strong localization of photons in certain disordered dielectric superlattices," Phys. Rev. Lett. 58, 24862489 (1987).

2. E. Yablonovitch, "Inhibited spontaneous emission in solidstate physics and electronics," Phys. Rev. Lett. 58, 2059$2062(1987)$

3. J. D. Joannopoulos, S. G. Johnson, J. N. Winn, and R. D.
Meade, Photonic Crystals: Molding the Flow of Light (Princeton U. Press, 2008).

4. M. Burger, S. J. Osher, and E. Yablonovitch, "Inverse problem techniques for the design of photonic crystals," IEICE Trans. Electron. E87C, 258-265 (2004).

5. J. S. Jensen and O. Sigmund have prepared a manuscript to be called "Topology optimization for nano-photonics-a review".

6. J. S. Jensen and O. Sigmund, "Systematic design of photonic crystal structures using topology optimization: Low-loss waveguide bends," Appl. Phys. Lett. 84, 2022-2024 (2004).

7. J. S. Jensen and O. Sigmund, "Topology optimization of photonic crystal structures: a high-bandwidth low-loss T-junction waveguide,” J. Opt. Soc. Am. B 22, 1191-1198 (2005).

8. W. R. Frei, D. A. Tortorelli, and H. T. Johnson, "Topology optimization of a photonic crystal waveguide termination to maximize directional emission," Appl. Phys. Lett. 86, 111114 (2005).

9. P. I. Borel, A. Harpoth, L. H. Frandsen, M. Kristensen, P. Shi, J. S. Jensen, and O. Sigmund, "Topology optimization and fabrication of photonic crystal structures," Opt. Express 12, 1996-2001 (2004).

10. L. H. Frandsen, A. Harpoth, P. I. Borel, M. Kristensen, J. S. Jensen, and O. Sigmund, "Broadband photonic crystal waveguide 60 degrees bend obtained utilizing topology optimization," Opt. Express 12, 5916-5921 (2004).

11. P. I. Borel, L. H. Frandsen, A. Harpoth, M. Kristensen, J. S. Jensen, and O. Sigmund, "Topology optimised broadband photonic crystal Y-splitter," Electron. Lett. 41, 69-71 (2005).

12. J. S. Jensen, O. Sigmund, L. H. Frandsen, P. I. Borel, A. Harpoth, and M. Kristensen, "Topology design and fabrication of an efficient double 90(circle) photonic crystal waveguide bend," IEEE Photon. Technol. Lett. 17, 1202-1204 (2005).

13. T. Nomura, K. Sato, K. Taguchi, T. Kashiwa, and S. Nishiwaki, "Structural topology optimization for the design of broadband dielectric resonator antennas using the finite difference time domain technique," Int. J. Numer. Methods Eng. 71, 1261-1296 (2007).

14. J. Dahl, J. S. Jensen, and O. Sigmund, "Topology optimization for transient wave propagation problems in one dimension design of filters and pulse modulators," Struct. Multidiscip. Optim. 36, 585-595 (2008).

15. L. R. Yang, A. V. Lavrinenko, J. M. Hvam, and O. Sigmund, "Design of one-dimensional optical pulse-shaping filters by time-domain topology optimization," Appl. Phys. Lett. 95, 261101 (2009).

16. J. S. Jensen, "Space-time topology optimization for onedimensional wave propagation," Int. J. Numer. Methods Eng. 198, 705-715 (2009).

17. J. Jin and D. J. Riley, Finite Element Analysis of Antennas and Arrays (Wiley, 2007).

18. D. Englund, I. Fushman, and J. Vuckovic, "General recipe for designing photonic crystal cavities," Opt. Express 13, 5961-5975 (2005)

19. W. R. Frei, H. T. Johnson, and K. D. Choquette, "Optimization of a single defect photonic crystal laser cavity," J. Appl Phys. 103, 033102 (2008).

20. Y. Akahane, M. Mochizuki, T. Asano, Y. Tanaka, and S. Noda, "Design of a channel drop filter by using a donor-type cavity with high-quality factor in a two-dimensional photonic crystal slab," Appl. Phys. Lett. 82, 1341-1343 (2003).

21. C. J. M. Smith, R. M. De la Rue, M. Rattier, S. Olivier, H. Benisty, C. Weisbuch, T. F. Krauss, R. Houdre, and U. Oesterle, "Coupled guide and cavity in a two-dimensional photonic crystal,” Appl. Phys. Lett. 78, 1487-1489 (2001).

22. G. H. Kim, Y. H. Lee, A. Shinya, and M. Notomi, "Coupling of small, low-loss hexapole mode with photonic crystal slab waveguide mode," Opt. Express 12, 6624-6631 (2004).

23. M. P. Bendsøe and O. Sigmund, Topology Optimization: Theory, Methods, and Applications, 2nd ed. (Springer Verlag, 2004).

24. H. A. Haus, Waves and Fields in Optoelectronics (PrenticeHall, 1984) 
25. A. F. Oskooi, L. Zhang, Y. Avniel, and S. G. Johnson, "The failure of perfectly matched layers, and towards their redemption by adiabatic absorbers," Opt. Express 16, 1137611392 (2008)

26. M. Koshiba, Y. Tsuji, and S. Sasaki, "High-performance absorbing boundary conditions for photonic crystal waveguide simulations," IEEE Microw. Wirel. Compon. Lett. 11, 152154 (2001).

27. S. Silver, Microwave Antenna Theory and Design (McGrawHill, 1949).

28. B. Yue and M. N. Guddati, "Dispersion-reducing finite elements for transient acoustics," J. Acoust. Soc. Am. 118, 2132-2141 (2005).

29. D. A. Tortorelli and P. Michaleris, "Design sensitivity analysis: overview and review," Inverse Probl. Eng. 1, 71-105 (1994)

30. Y. S. Chung, C. Cheon, I. H. Park, and S. Y. Hahn, "Optimal shape design of microwave device using FDTD and design sensitivity analysis," IEEE Trans. Microwave Theory Tech. 48, 2289-2296 (2000).

31. D. A. Tortorelli and R. B. Haber, "First-order design sensitivities for transient conduction problems by an adjoint method," Int. J. Numer. Methods Eng. 28, 733-752 (1989).

32. J. D. Jackson, Classical Electrodynamics (Wiley, 1999).

33. K. Svanberg, "A class of globally convergent optimization methods based on conservative convex separable approximations," SIAM J. Optim. 12, 555-573 (2002).

34. O. Sigmund, "Morphology-based black and white filters for topology optimization," Struct. Multidiscip. Optim. 33, 401424 (2007).

35. J. K. Guest, "Topology optimization with multiple phase projection," Comput. Methods Appl. Mech. Eng. 199, 123135 (2009).

36. http://www.esf.org/euryi.

37. http://www.topopt.dtu.dk. 